\title{
Erythropoietin Modulates Cerebral and Serum Degradation Products from Excess Calpain Activation following Prenatal Hypoxia-Ischemia
}

\author{
Lauren L. Jantzie ${ }^{a, b, d, ~ e ~ J e s s e ~ L . ~ W i n e r ~}{ }^{a} \quad$ Christopher J. Corbett $^{a}$ \\ Shenandoah Robinson ${ }^{a-c}$ \\ Departments of a Neurosurgery and ${ }^{b}$ Neurology and ${ }^{\mathrm{C}}$ Kirby Center for Neurobiology, Boston Children's Hospital \\ and Harvard Medical School, Boston, Mass., and Departments of ${ }^{\mathrm{d}}$ Pediatrics and ${ }^{\mathrm{e}}$ Neurosciences, University of \\ New Mexico, Albuquerque, N. Mex., USA
}

\section{Key Words}

Brain injury - Calpain - Erythropoietin · KCC2 - Myelin .

Neurofilament $\cdot$ Preterm birth $\cdot$ Prematurity $\cdot$ Serum

biomarker $\cdot$ Spectrin

\begin{abstract}
Preterm infants suffer central nervous system (CNS) injury from hypoxia-ischemia and inflammation - termed encephalopathy of prematurity. Mature CNS injury activates caspase and calpain proteases. Erythropoietin (EPO) limits apoptosis mediated by activated caspases, but its role in modulating calpain activation has not yet been investigated extensively following injury to the developing CNS. We hypothesized that excess calpain activation degrades developmentally regulated molecules essential for CNS circuit formation, myelination and axon integrity, including neuronal potassium-chloride co-transporter (KCC2), myelin basic protein (MBP) and phosphorylated neurofilament (pNF), respectively. Further, we predicted that post-injury EPO treatment could mitigate CNS calpain-mediated degradation. Using prenatal transient systemic hypoxia-ischemia (TSHI) in rats to mimic CNS injury from extreme preterm birth, and postnatal EPO treatment with a clinically relevant dosing regimen, we found sustained postnatal excess cortical calpain activation following prenatal TSHI, as shown by the cleavage
\end{abstract}

of alpha II-spectrin (all-spectrin) into 145-kDa all-spectrin degradation products (all-SDPs) and p35 into p25. Postnatal expression of the endogenous calpain inhibitor calpastatin was also reduced following prenatal TSHI. Calpain substrate expression following TSHI, including cortical KCC2, MBP and $\mathrm{NF}$, was modulated by postnatal EPO treatment. Calpain activation was reflected in serum levels of all-SDPs and KCC2 fragments, and notably, EPO treatment also modulated KCC2 fragment levels. Together, these data indicate that excess calpain activity contributes to the pathogenesis of encephalopathy of prematurity. Serum biomarkers of calpain activation may detect ongoing cerebral injury and responsiveness to EPO or similar neuroprotective strategies.

(c) 2015 S. Karger AG, Basel

\section{Introduction}

Preterm birth alters the microenvironment in the developing central nervous system (CNS) over a sustained period of maturation before, during and after birth. The cumulative injury causes an amalgam of damage termed encephalopathy of prematurity [1]. Improved identification and refinement of therapeutic interventions depends upon a precise understanding of the mechanisms and duration of injury and potential treatment targets. For ex-

\section{KARGER}

E-Mail karger@karger.com

www.karger.com/dne
(C) 2015 S. Karger AG, Basel

0378-5866/15/0381-0015\$39.50/0
Shenandoah Robinson, MD

Department of Neurosurgery, Hunnewell 2 Boston Children's Hospital

300 Longwood Ave., Boston, MA 02115 (USA)

E-Mail shenandoah.robinson@ childrens.harvard.edu 
ample, the emerging neurorestorative agent erythropoietin (EPO) is currently being administered in a clinical trial for extremely preterm infants born before 28 weeks estimated gestational age without adjustment of the dosing regimen to the type or severity of the initial CNS injury, the presence of cumulative secondary insults or the individual vulnerability and response of the patient to the CNS injury (PENUT trial, NCT01378273). Serum biomarkers that reflect the CNS injury and the potential for recovery offer a promising modality to stratify patients and guide the dosing of emerging interventions.

Two major mediators of CNS injury are caspase and calpain activation [2]. Apoptosis identified by cleaved caspase- 3 immunolabeling has been found in white matter lesions associated with preterm birth [3-5], and activated caspase- 3 has been found in the cerebrospinal fluid of preterm infants [6]. EPO is a known CNS antiapoptotic agent [7]. Using a rat model of prenatal transient systemic hypoxia-ischemia (TSHI) that mimics encephalopathy of prematurity observed in human infants who are born extremely preterm [8], postnatal EPO administered in a clinically relevant paradigm reduced injury-induced caspase- 3 activation and improved functional outcomes in adult rats [9]. However, the impact of EPO on calpain signaling in encephalopathy of prematurity has not been elucidated.

Calpains, calcium-activated proteases, have an active role in cellular homeostasis both during development and in the mature CNS by degrading proteins. Calpain activity is modulated in part by the endogenous calpain inhibitor calpastatin, and both calpain and calpastatin levels are developmentally regulated [10]. Appropriate calpain activity is essential for neurodevelopment, including learning and memory [11] and axon pruning [12]. In the CNS, calpain is quantified by measuring the degradation of full-length $(250 \mathrm{kDa})$ membrane-associated cytosolic protein alpha II-spectrin (aII-spectrin) into 150 - and $145-\mathrm{kDa}$ spectrin degradation products (aII-SDPs) [13]. By contrast, activated caspase cleaves aII-spectrin into $150-$ and $120-\mathrm{kDa}$ aII-SDPs [13]. In pathological neurodegenerative, demyelinating or posttraumatic processes in the mature CNS, excess calpain activity degrades molecules important for neurological function [14-17]. Previously, we have shown that prenatal TSHI induces excess NMDAR (N-methyl-D-aspartic acid receptor) activation and calcium-mediated calpain activation that limits potassium-chloride cotransporter (KCC2) expression in the hippocampal CA3 subfield, and that postnatal EPO treatment reverses excess calpain activation [18]. Here, we investigated the broader impact of excess calpain activation following prenatal HI injury.

In the present investigation, we examined the impact of calpain degradation in encephalopathy of prematurity in detail by determining the extent and time course of calpain degradation of molecules important to perinatal neurodevelopment. In addition, we investigated whether postinjury EPO treatment impacts the TSHI-induced loss of expression of molecules that reflect neurodevelopmental maturation. Specifically, we examined cortical expression levels of KCC2 oligomers, myelin basic protein (MBP) and the phosphorylated neurofilament/total neurofilament ( $\mathrm{pNF} / \mathrm{NF}$ ) ratio following prenatal injury and postnatal EPO treatment. First, we focused on these molecules because of their importance in neuronal signaling, myelination and axonal health during perinatal brain development, respectively. Second, these molecules were investigated because they are known calpain substrates [14, 17-21]. Finally, we sought to determine whether serum levels reflect the cerebral changes in calpain degradation and EPO treatment. We hypothesized that prenatal HI that mimics a component of encephalopathy of prematurity will cause excess CNS calpain activation, subsequent degradation of key neurodevelopmental molecules and associated signs of elevated calpain activity in serum. Moreover, we postulated that postnatal treatment with the neuroprotective agent EPO could modulate excess CNS calpain activity, and that this effect of EPO treatment on calpain activity would be reflected in the serum. We provide novel evidence that TSHI induces a marked elevation of calpain activity in the CNS during a critical point in brain development, and that postnatal EPO treatment attenuates calpain activity and preserves downstream targets essential to normal CNS maturation. Further, we show that aII-SDP and KCC2 fragments are detected in serum following injury and may prove useful as biomarkers of CNS injury and EPO efficacy.

\section{Materials and Methods}

\section{Prenatal TSHI}

The Boston Children's Hospital Institutional Animal Care and Use Committee approved all experimental protocols. Prenatal TSHI was performed on embryonic day 18 (E18) as previously described $[8,9]$. Briefly, timed pregnant Sprague-Dawley dams were purchased from Charles River Laboratories, and laparotomy was performed under isoflurane anesthesia on E18 with uterine artery occlusion for $60 \mathrm{~min}$. Shams underwent anesthesia and laparotomy without artery occlusion. Pups were born at term and remained with their dams until weaning on postnatal day 21 (P21). Rats of both sexes were used in all experiments.
16

Dev Neurosci 2016;38:15-26 DOI: $10.1159 / 000441024$
Jantzie/Winer/Corbett/Robinson 


\section{EPO Treatment}

After birth, TSHI pups were randomly assigned to receive intraperitoneal EPO (2,000 U/kg/dose; R\&D Systems) or vehicle (sterile saline) administered daily from P1 to P5. We have previously found that this dosing regimen results in sustained functional improvement in adult rats following prenatal TSHI $[9,22]$, and have documented that no significant knowledge was gained from a sham EPO cohort [9].

\section{Tissue and Serum Collection}

For immunohistochemistry at P2, brains were drop-fixed in $4 \%$ paraformaldehyde. After fixation, the brains were immersed in $30 \%$ sucrose and processed for immunohistochemistry as below. For immunoblotting, cortical samples were collected from at least two separate litters for each group and snap-frozen at $-80^{\circ} \mathrm{C}$. Specific numbers of samples analyzed in each experiment are provided. For P5 and younger, cerebral cortical samples were collected from the frontal lobes. Samples from two P11 brains were microdissected and pooled to provide an adequate final sample for the cortical membrane preparations for the KCC2 analyses and for the white matter MBP and NF analyses. Microdissected samples from single animals were used for P28. For serum analyses, blood was collected from each pup and centrifuged at $6,000 \mathrm{~g}$ for $15 \mathrm{~min}$, and serum was removed and stored in aliquots at $-80^{\circ} \mathrm{C}$ for later analysis. Care was taken to avoid repeated freeze-thaw cycles.

\section{Immunoblotting}

Western blots were performed as previously described [18, 22]. For analyses of cerebral tissue, samples were sonicated, and whole cell and membrane fractions were separated via differential centrifugation. Bradford protein assays were performed per manufacturer specifications (Bio-Rad, Hercules, Calif., USA). Proteins were separated on $4-20 \%$ Tris $\mathrm{HCl}$ gels, with the exception of MBP Western blots in which proteins were separated on $4-10 \%$ Bis Tris XT gels (all Bio-Rad). In the experiment to better delineate aIISDP, 7.5\% Tris $\mathrm{HCl}$ gels were used for optimal separation in the 120 - to $150-\mathrm{kDa}$ range. For cerebral tissue, $30 \mu \mathrm{g}$ was loaded per lane and after electrophoretic separation the proteins were transferred to PVDF (polyvinylidene fluoride) membranes for incubation with antibodies. The following primary antibodies were used: aII-spectrin (Santa Cruz Biotechnology, Dallas, Tex., USA; 1:100), calpastatin (Santa Cruz Biotechnology; 1:100), KCC2 for $90-\mathrm{kDa}$ N-terminal fragment (Santa Cruz Biotechnology; 1:100), KCC2 oligomers (Millipore, Billerica, Mass., USA; 1:1,000), MBP (Millipore; 1:500), NF (Millipore; 1:1,000) and pNF (SMI-312; Covance, Dedham, Mass., USA; 1:500). For loading controls, $\beta$-tubulin (Covance) was used for membrane fractions, and $\beta$-actin (Sigma-Aldrich, St. Louis, Mo., USA) for whole cell preparations. Images were developed with chemiluminescence, imaged (LAS 4000; GE Healthcare, Piscataway, N.J., USA) and quantified (ImageQuant; GE Healthcare). Serum samples were diluted, protein assays were performed, and $120 \mu \mathrm{g}$ of serum protein was loaded in each lane. Coomassie staining was used as the loading control for serum.

\section{Immunohistochemistry}

Frozen coronal sections $(20 \mu \mathrm{m})$ were cut on a cryostat (Leica). Sections were incubated sequentially with $0.3 \%$ hydrogen peroxide, blocking agent consisting of $10 \%$ goat serum in phosphatebuffered saline, primary antibodies in blocking solution with $0.1 \%$ Triton overnight at $4{ }^{\circ} \mathrm{C}$, rinsed, incubated with appropriate sec-

EPO Modulates Calpain Activity

following Prenatal HI ondary antibodies in blocking solution for $1 \mathrm{~h}$, incubated with VECTASTAIN (Vector Laboratories), visualized with diaminobenzidine, dehydrated and mounted. Primary antibodies used were anti-calpain (Santa Cruz Biotechnology; 1:250) and anti-calpastatin (Santa Cruz Biotechnology; 1:250). Using a Leica microscope with bright-field illumination, representative images were photographed at $20 \times$ magnification.

\section{Calpain Digestion Assay}

To confirm calpain-mediated degradation of specific CNS molecules, calpain digestion of sham samples was performed at P7 and P11 $[23,24]$. Briefly, snap-frozen tissue was thawed and lysed in lysis buffer containing $50 \mathrm{~mm}$ Tris- $\mathrm{HCl}$ (pH 7.4), $5 \mathrm{~mm}$ EDTA (ethylene diaminetetraacetic acid), 5 mM EGTA (ethylene glycol tetraacetic acid), $1 \%$ Triton X-100 and 1 mM DTT (dithiothreitol) for $90 \mathrm{~min}$ at $4^{\circ} \mathrm{C}$. After centrifugation $(3,000 \mathrm{~g}$ for $5 \mathrm{~min}$ ), protein assays were performed. Samples $(100 \mu \mathrm{g})$ were digested with human erythrocyte calpain 1 (Calbiochem, Billerica, Mass., USA; 1:200) in a buffer containing $100 \mathrm{~mm}$ Tris- $\mathrm{Cl}$ (pH 7.4), $20 \mathrm{~mm}$ DTT and $1 \mathrm{mM} \mathrm{CaCl}_{2}$ for $30 \mathrm{~min}$ at room temperature. The reaction was terminated by the addition of $8 \times$ sample loading buffer containing $0.25 \mathrm{M}$ Tris (pH 6.8), $0.2 \mathrm{M}$ DTT, $8 \%$ SDS, $0.2 \%$ bromophenol blue and $20 \%$ glycerol in distilled water. Proteins were separated by electrophoresis, transferred, probed with target antibodies and visualized as above.

\section{Statistical Analyses}

For analysis of two groups (sham and TSHI) at one time point, differences were compared using two-tailed Student's t test with unequal variance. For two groups over multiple ages and for analysis of the sham, TSHI-vehicle (TSHI-veh) and TSHI-EPO groups, two-way ANOVA was performed. Bonferroni's post hoc correction was used for multiple comparisons. Analyses were performed using SPSS 21 (IBM, Armonk, N.Y., USA), with $\mathrm{p}<0.05$ considered significant.

\section{Results}

\section{Cerebral Cortical Calpain Activity Is Elevated following Prenatal TSHI}

A summary of the experimental design and primary end points with complete quantification is illustrated in figure 1 . To determine the contribution of calpain and caspase activity to aII-spectrin degradation in our in vivo model, we performed a calpain digestion assay to identify primary aII-SDPs. Specifically, to resolve the primary aII-SDPs of 120, 145 and $150 \mathrm{kDa}$, we digested P7 sham control cortex with calpain in the presence of calcium, as previously reported [23]. In a calpain digestion assay of cortical aII-spectrin, $\alpha$ II-SDPs appeared in the sham P7 cortex at 145 and $150 \mathrm{kDa}$, with the $145-\mathrm{kDa}$ band much more prominent than the $150-\mathrm{kDa}$ band (fig. 2a). By contrast, only a faint band was present at $120 \mathrm{kDa}$, suggesting relatively minimal caspase activity. Similarly, to confirm the degradation of a second calpain target, we also exam- 


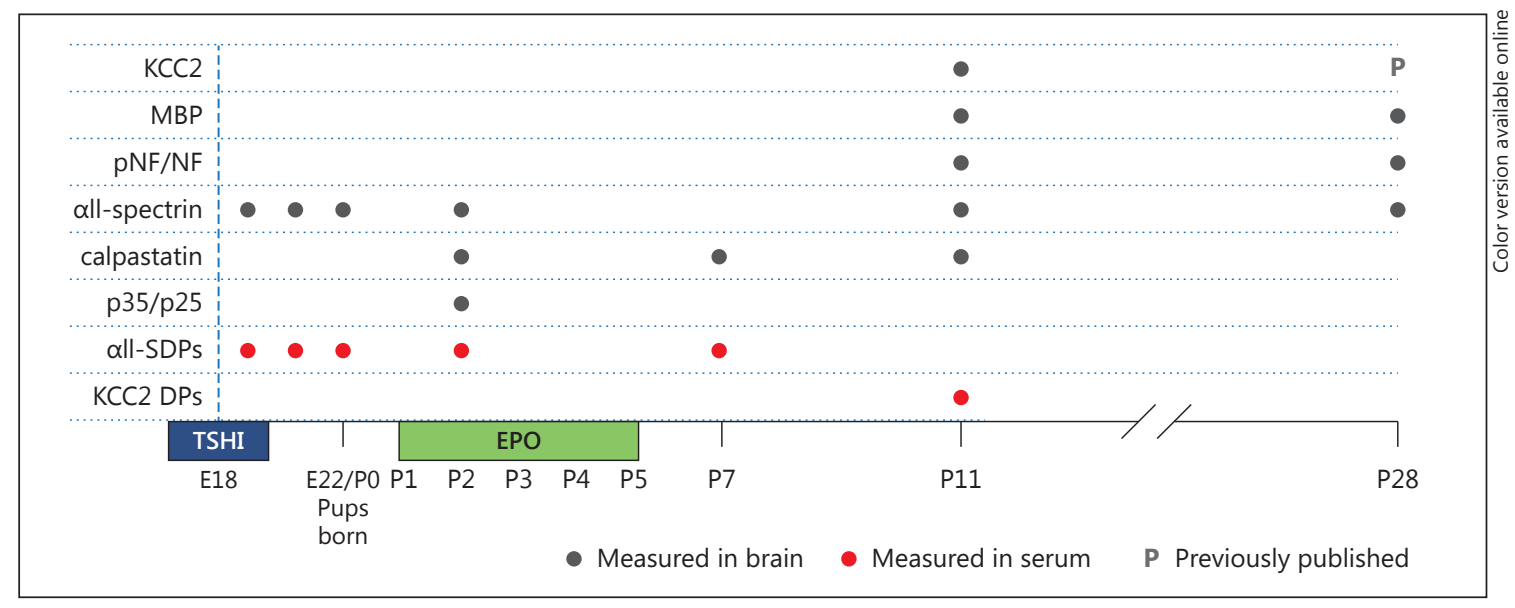

Fig. 1. Summary of experimental design and major data points. TSHI was performed on E18 via laparotomy with 60 min of uterine artery occlusion. Pups were born at term (E22). TSHI pups were randomized to receive either EPO $(2,000 \mathrm{U} / \mathrm{kg} / \mathrm{dose})$ or vehicle daily from P1 to P5. Red dots (color in online version only) indicate when samples were collected from serum for quantification, while gray dots represent samples collected from frontal lobes (prior to P5) and cortex and white matter (older than P5) for quantification.

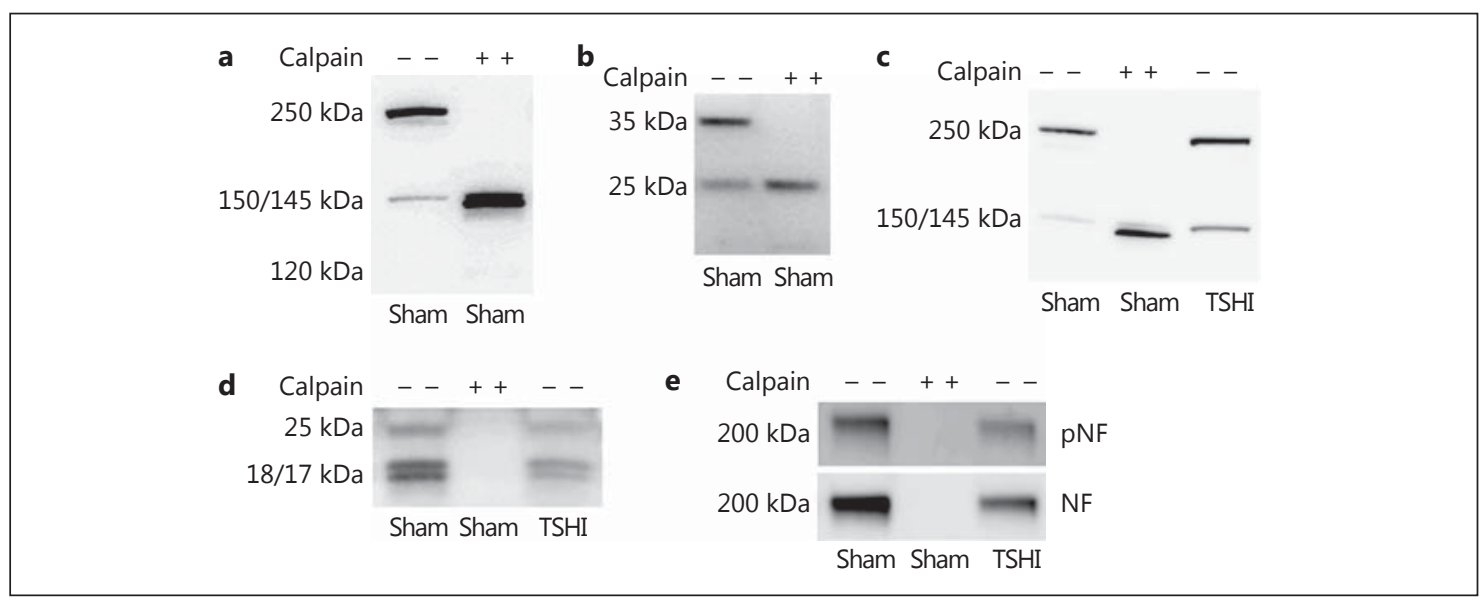

Fig. 2. Calpain digestions of cortex and white matter. a P7 aIISDPs. Representative calpain digestion of P7 sham cortex incubated with aII-spectrin antibodies reveals a primary band at $250 \mathrm{kDa}$ and a fainter band at $150 \mathrm{kDa}$. In the presence of calcium-activated $\mu$-calpain, the aII-spectrin is digested into a faint $150-\mathrm{kDa}$, a heavy $145-\mathrm{kDa}$ and a very faint $120-\mathrm{kDa}$ band. b P7 p35/25. Sham P7 cortex digested with calpain shows elimination of the p35 band, with a corresponding increase in the p25 fragment. c P11 aII-SDPs. Calpain digestion of P11 sham cortex assayed alongside P11 TSHI cor-

ined the cleavage of known calpain substrate protein 35 (p35) into a p25 fragment [25]. As expected, in the sham P7 cortex, we found an increase in the p25 fragment in calpain-digested samples in the presence of calcium (fig. 2b). Next, we compared the pattern of aII-SDPs in a calpain digestion of the P11 sham cortex with the aII- tex probed with $\alpha \mathrm{II}$-spectrin shows a band at $150 / 145 \mathrm{kDa}$ that is similar to a band present in sham cortex digested with activated calpain but not present in sham cortex without calpain digestion. d P11 MBP. Calpain digestion of P11 sham white matter in the presence of calcium eliminates MBP, while P11 TSHI white matter assayed in an adjacent lane shows reduced expression. e P11 pNF/ NF. Calpain digestion of P11 sham white matter in the presence of calcium eliminates pNF and NF, while P11 TSHI white matter assayed in an adjacent lane shows reduced pNF and NF expression. 
Fig. 3. Excess calpain activity is induced by prenatal TSHI and modulated by EPO treatment. a aII-spectrin ratio. Representative Western blot of aII-SDPs and ratio of cleaved to full-length cerebral aII-SDPs at $6 \mathrm{~h}(\mathrm{E} 18+6), 24 \mathrm{~h}(\mathrm{E} 19), 96 \mathrm{~h}(\mathrm{P} 0)$ and 6 days (P2) after prenatal TSHI compared to sham. A significant increase in aII-SDPs is present at $\mathrm{P} 2$. b $\mathrm{P} 2 \mathrm{p} 35 / 25$ ratio. The ratio of p25/p35 is increased at P2 following prenatal TSHI. c P11 aII-spectrin ratio. Following prenatal TSHI, the ratio of cleaved to full-length $\alpha \mathrm{II}-\mathrm{SDP}$ at P11 is elevated compared to sham. Postnatal treatment with EPO normalizes the ratio to sham levels (two-way ANOVA with Bonferroni correction). ${ }^{*} \mathrm{p}<0.05,{ }^{* *} \mathrm{p}<0.01$.
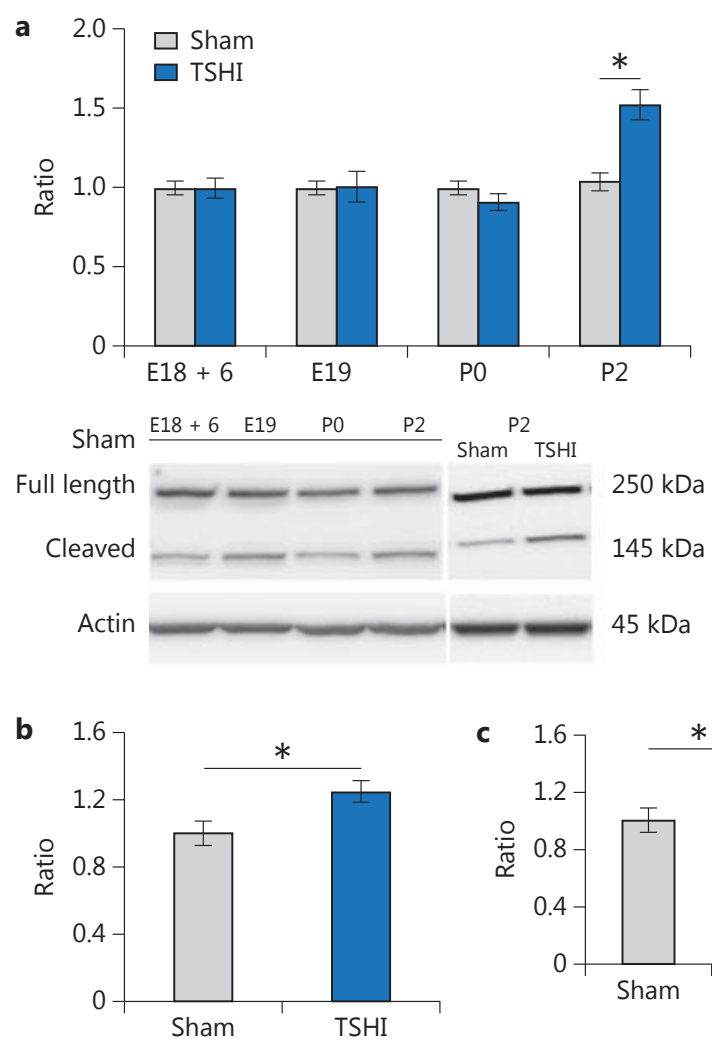

C
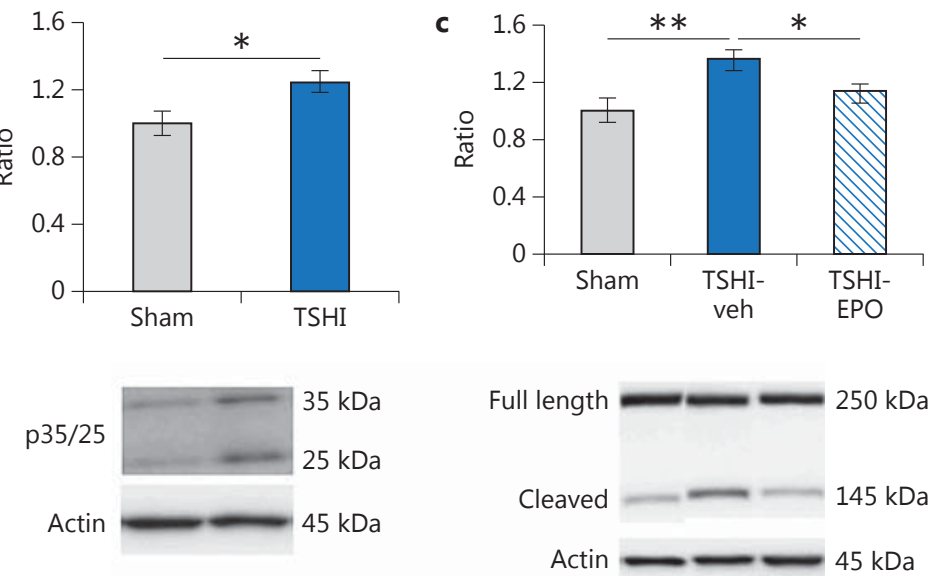

to determine whether excess calpain activity would affect calpain targets in white matter such as MBP, pNF and NF. Thus, we performed calpain digestion assays to confirm that calpain degrades these molecules in developing white matter. Indeed, the digestion of P11 sham white matter samples with calpain in the presence of calcium degraded MBP (fig. 2d), pNF and NF (fig. 2e). These patterns were compared to representative P11 TSHI-veh white matter samples. Together, these data demonstrate that our assays could detect calpain activity and that calpain degrades molecules central to gray and white matter development, including MBP and pNF/NF. Thus, we proceeded with our investigation and quantitation of excess calpain activity in this model of prenatal TSHI.

To clarify the time course of calpain activity in the cerebral cortex following prenatal TSHI, the ratios of cleaved $(145 \mathrm{kDa})$ to full-length $(250 \mathrm{kDa})$ aII-spectrin were analyzed in the TSHI cortex at multiple early time

EPO Modulates Calpain Activity

following Prenatal HI points after prenatal TSHI (E18 +6 h, E19, P0 and P2). No differences after prenatal injury were evident until P2 ( $\mathrm{n}=12-15$, two-way ANOVA, $\mathrm{p}=0.007$; fig. 3a), approximately 6 days after the insult on E18, suggesting the delayed appearance of excess calpain degradation of aIIspectrin and thus the delayed onset of excess calpain activity within the CNS. To confirm that excess cerebral calpain activity was present following prenatal TSHI, we also quantified the degradation of p35 to p25 at P2 and found more $\mathrm{p} 25$ in the TSHI cortex $(\mathrm{n}=11)$ than in the sham cortex $(n=10, p=0.026$; fig. $3 b)$. Together, these findings show that excess CNS calpain activity is initially detected at $\mathrm{P} 2$ in the CNS in this model.

\section{Postinjury EPO Treatment Limits Calpain Degradation}

EPO treatment in vivo has been implicated in reducing calpain activity in other types of mature CNS insults [26], 
Fig. 4. Prenatal TSHI reduces calpastatin expression. $\mathrm{WM}=$ White matter. a At P2 immunolabeling with calpastatin antibodies shows loss of prominent cell labeling in the white matter, subplate and cortex following TSHI compared to sham. By contrast, immunolabeling with $\mu$-calpain antibodies is increased after TSHI. Scale bar = $20 \mu \mathrm{m}$. b P7 calpastatin. Cortical calpastatin expression is reduced at $\mathrm{P} 7$ following prenatal TSHI compared to sham. c P11 calpastatin. Following prenatal TSHI, calpastatin expression is reduced at $\mathrm{P} 11 \mathrm{com}$ pared to sham. Postnatal treatment with EPO restores calpastatin expression to sham levels (two-way ANOVA with Bonferroni correction). ${ }^{*} \mathrm{p}<0.05$.

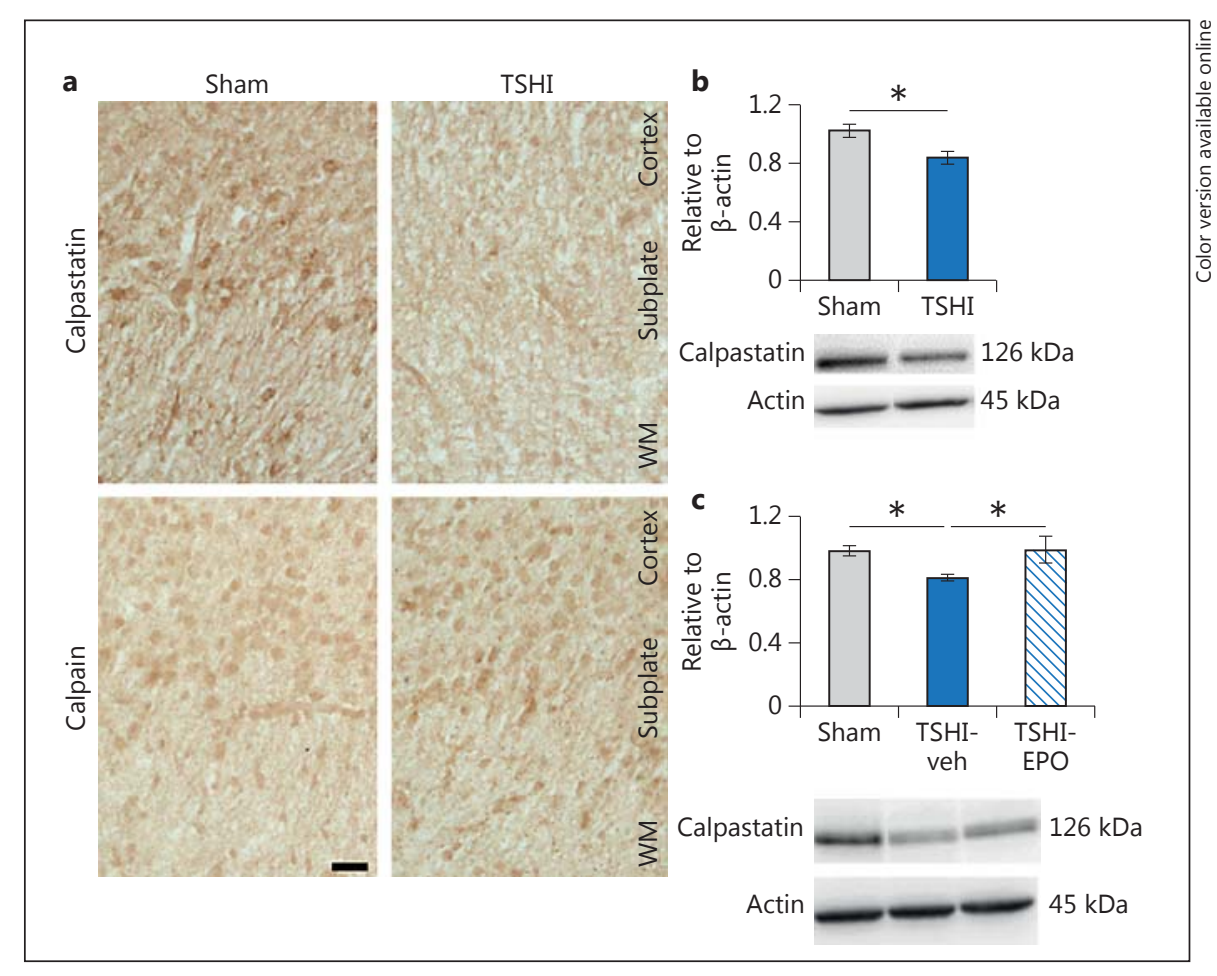

but this mechanism has not previously been investigated in impaired CNS development from very preterm birth. To test whether postinjury postnatal EPO treatment alters calpain activity in this rat model of encephalopathy of prematurity, we analyzed cortical cleaved/full-length aII-SDP ratios at P11 and P28. Ratios of cleaved/fulllength aII-SDPs were elevated in the P11 TSHI-veh cortex $(\mathrm{n}=14)$ compared to shams $(\mathrm{n}=22$, two-way ANOVA, $\mathrm{p}=0.002)$ or TSHI-EPO cortex $(\mathrm{n}=14, \mathrm{p}=0.016$; fig. 3c), and the differences resolved by P28 (data not shown). These data show that EPO can modulate CNS calpain activity in vivo following early injury to the developing brain.

\section{TSHI Alters the Balance between Calpain and Calpastatin}

Both calpain and the endogenous calpain inhibitor calpastatin are developmentally regulated [10], and calpain activity reflects the balance of activated calpain and calpastatin. Calpastatin immunolabeling in the cortex, subplate and white matter at P2 was reduced in TSHI brains compared to shams, with the most prominent difference evident in the subplate (fig. 4a). By contrast, a mild increase in calpain expression was present in the white matter at P2. Cerebral calpastatin levels were quantified using immunoblots. Calpastatin levels were signifi- cantly reduced at $\mathrm{P} 7(\mathrm{n}=12$, $\mathrm{t}$ test, $\mathrm{p}=0.007$; fig. $4 \mathrm{~b})$ but not earlier at P2 (data not shown). Similarly, calpastatin levels were reduced at P11 in TSHI-veh brains $(\mathrm{n}=12)$ compared to shams $(\mathrm{n}=12$, two-way ANOVA, $\mathrm{p}=0.024)$ and restored in TSHI-EPO rats $(\mathrm{n}=12, \mathrm{p}=0.03$; fig. $4 \mathrm{c})$. Together, these data show that the cerebral cortical aIISDP ratio is elevated and calpastatin expression is reduced with late-gestation CNS injury, which is consistent with excess calpain activation. Moreover, both the CNS aII-SDP ratio and calpastatin expression are responsive to postnatal EPO treatment.

\section{Postinjury EPO Treatment Limits Calpain Degradation of Cortical KCC2 and White Matter $M B P$ and NF}

We hypothesized that in addition to injury-induced calpain degradation of aII-spectrin, excess CNS calpain activity caused by prenatal TSHI would also degrade other known calpain substrates, including molecules essential to neurological development and function. Calpain cleaves the neuronal potassium-chloride co-transporter KCC2 into $90-\mathrm{kDa} \mathrm{N}$-terminal and $30-\mathrm{kDa}$ C-terminal fragments that are labeled with $\mathrm{N}$ - and C-terminal-specific antibodies $[19,20]$, and calpain degradation is the primary cause of reduced KCC2 levels present in the CA3 region following prenatal TSHI [18]. Here, we examined 


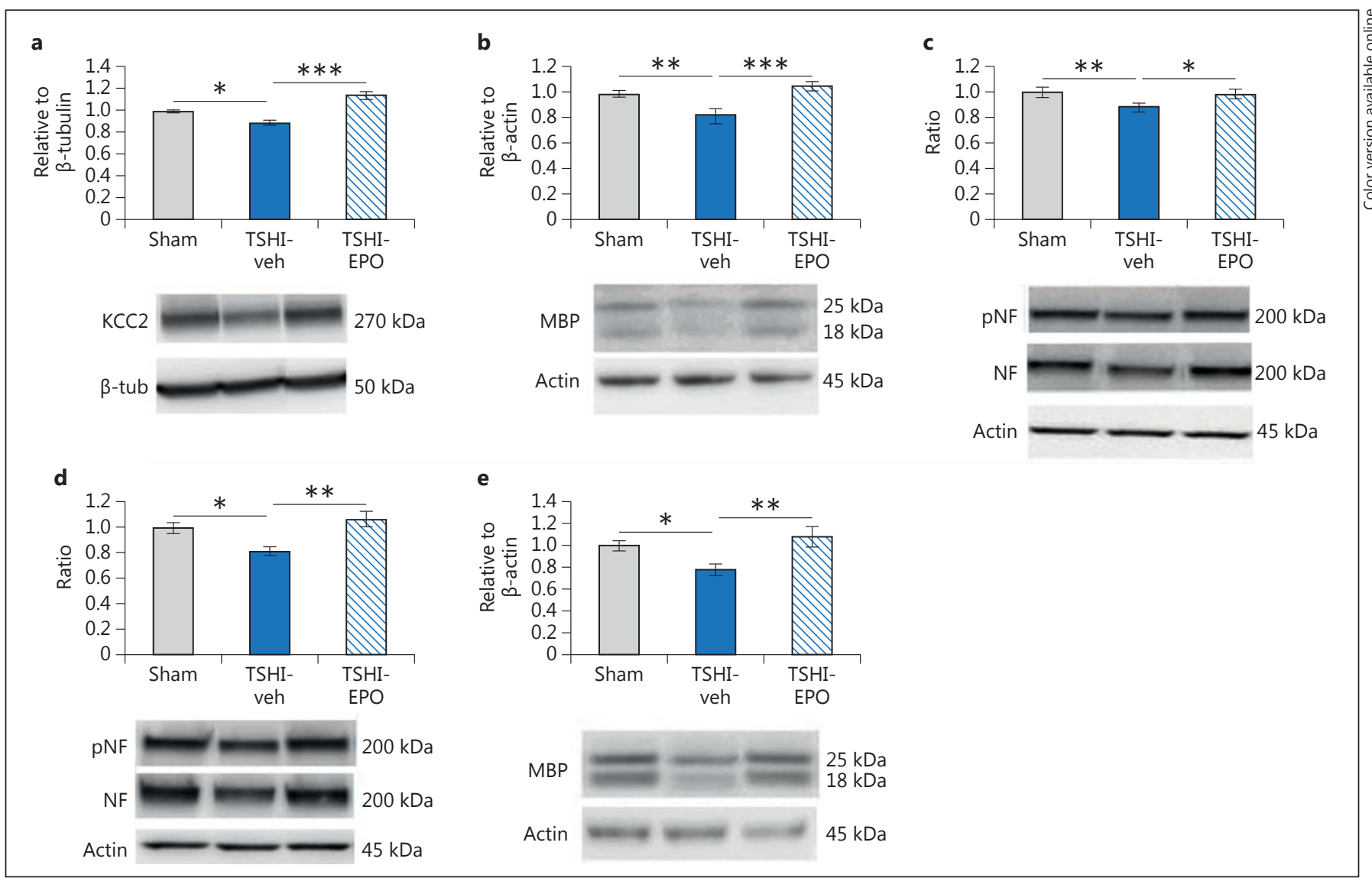

Fig. 5. Calpain degrades molecules essential for neurodevelopment, including KCC2, MBP and NF. a P11 cortex KCC2. At P11 KCC2 oligomer expression is reduced following prenatal TSHI, and KCC2 levels are restored following postnatal EPO treatment. b P11 white matter MBP. At P11 in the midst of myelin development, MBP expression is reduced following prenatal TSHI and normalizes following EPO treatment. c P11 white matter pNF/NF ratio. Similarly, at $\mathrm{P} 11$ the ratio of $\mathrm{pNF} / \mathrm{NF}$ is lower following pre- natal TSHI and restored following EPO treatment. d P28 white matter $\mathrm{pNF} / \mathrm{NF}$ ratio. At $\mathrm{P} 28$ in juvenile white matter, the $\mathrm{pNF} / \mathrm{NF}$ ratio at P28 is also reduced following prenatal TSHI and restored with postnatal EPO treatment. e P28 white matter MBP. Likewise, the white matter MBP levels remain lower following prenatal TSHI and normalize with EPO treatment (two-way ANOVA with Bonferroni correction). ${ }^{*} \mathrm{p}<0.05,{ }^{* *} \mathrm{p}<0.01,{ }^{* * *} \mathrm{p} \leq 0.001$. cerebral cortical levels of KCC2 following prenatal TSHI and postnatal EPO treatment. Compared to shams ( $\mathrm{n}=$ 12), P11 cortical levels of oligomeric KCC2 were decreased in prenatal TSHI-veh rats $(\mathrm{n}=12$, two-way ANO$\mathrm{VA}, \mathrm{p}=0.024)$ and improved with EPO treatment $(\mathrm{n}=$ 12 , p $<0.001$; fig. 5a). Consistent with our previous studies that showed a loss of KCC2 expression in the CA3 subfield [18] and cortical layer IV following prenatal TSHI [22], these results also suggest that postnatal EPO treatment can mitigate the loss of cerebral cortical KCC2 expression induced by prenatal TSHI.

Next, to quantify the loss of MBP and NF following prenatal TSHI, we assayed MBP levels and $\mathrm{pNF} / \mathrm{NF}$ ratios, respectively. At P11 MBP levels were reduced in
TSHI-veh samples $(n=12)$ compared to shams $(n=18$, two-way ANOVA, $\mathrm{p}=0.003$ ) and TSHI-EPO brains $(\mathrm{n}=$ 20, $\mathrm{p}<0.001$; fig. 5b). At P11 samples from TSHI-veh brains $(\mathrm{n}=26)$ assayed for $\mathrm{pNF} / \mathrm{NF}$ also showed lower levels than shams $(\mathrm{n}=24, \mathrm{p}=0.007)$ or TSHI-EPO brains ( $\mathrm{n}=22, \mathrm{p}=0.015$; fig. $5 \mathrm{c}$ ). To determine whether the loss of MBP and NF persisted in juvenile brains, we examined $\mathrm{MBP}$ and $\mathrm{pNF} / \mathrm{NF}$ levels at P28. Similar to P11, the pNF/ NF ratio was reduced at $\mathrm{P} 28$ in TSHI-veh rats $(\mathrm{n}=10)$ compared to shams $(\mathrm{n}=9, \mathrm{p}=0.011)$ and TSHI-EPO brains ( $\mathrm{n}=7, \mathrm{p}=0.001$; fig. $5 \mathrm{~d})$. MBP levels from P28 TSHI-veh rats $(\mathrm{n}=13)$ were also lower than shams $(\mathrm{n}=$ $12, \mathrm{p}=0.038)$ or TSHI-EPO rats $(\mathrm{n}=8, \mathrm{p}=0.009$; fig. $5 \mathrm{e})$. Together, these results show that MBP and pNF/NF levels 


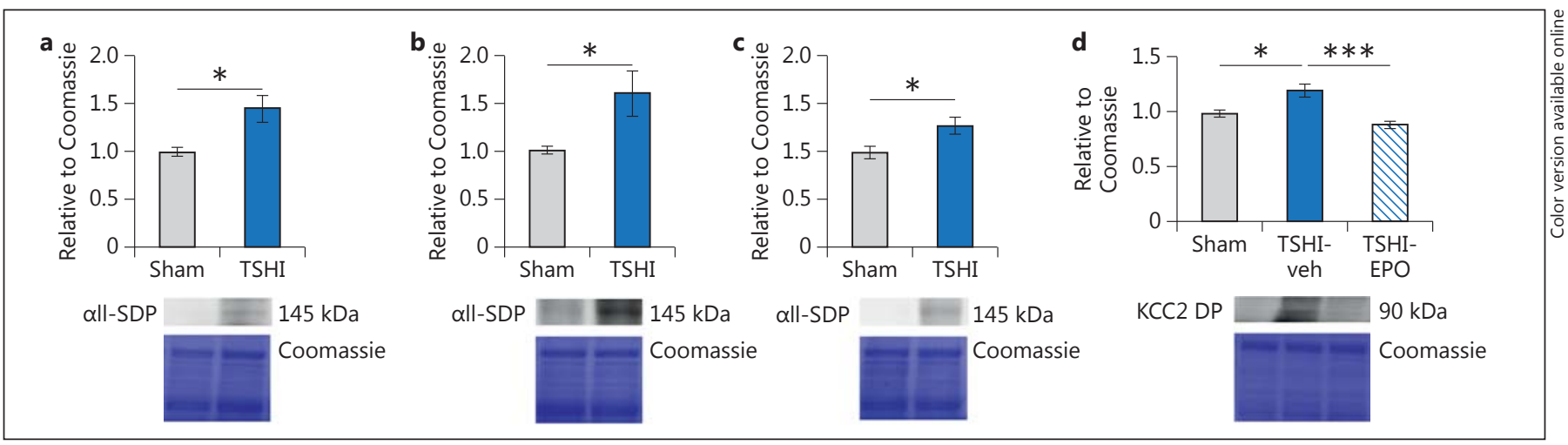

Fig. 6. Calpain degradation products are present in serum. Serum levels of $145-\mathrm{kDa}$ aII-SDP are elevated at E19 (a), P0 (b) and P2 (c; unpaired two-tailed t test). Coomassie staining was used for the loading control. d P11 serum KCC2 fragment. At P11 serum levels of the $90-\mathrm{kDa} \mathrm{KCC} 2$ calpain fragment are elevated following prenatal TSHI and normalized with postnatal EPO treatment (twoway ANOVA with Bonferroni correction). ${ }^{*} \mathrm{p}<0.05,{ }^{* * *} \mathrm{p} \leq 0.001$. are reduced following prenatal TSHI for a sustained, critical period of postnatal CNS development, probably due in part to calpain degradation, and that these levels are responsive to postnatal EPO treatment.

\section{Serum $\alpha$ II-SDP Levels following Prenatal TSHI}

For selected neuromolecules, serum levels reflect CNS levels, and the presence of CNS molecules in the serum is a biomarker of brain injury. As cerebral cortical aII-SDP levels are elevated following prenatal TSHI, we investigated the presence of serum $145-\mathrm{kDa}$ aII-SDPs following prenatal TSHI along the same time course. Serum 145$\mathrm{kDa}$ aII-SDP levels were elevated in TSHI-veh rats $(\mathrm{n}=$ 10) at E19, $24 \mathrm{~h}$ after the E18 injury, compared to shams $(\mathrm{n}=15$, $\mathrm{t}$ test, $\mathrm{p}=0.012$; fig. $6 \mathrm{a})$. Serum $\alpha I I-S D P s$ remained elevated in serum at P0 following TSHI compared to shams (both: $\mathrm{n}=11, \mathrm{p}=0.037$; fig. $6 \mathrm{~b}$ ) and at $\mathrm{P} 2$ (sham: $\mathrm{n}=15$, TSHI: $\mathrm{n}=10, \mathrm{p}=0.022$; fig. $6 \mathrm{c}$ ). By P7, however, the difference was no longer significant (sham: $\mathrm{n}=11$, TSHI: $\mathrm{n}=8$, data not shown). Together, these results suggest that the serum aII-SDP levels indicative of excess calpain activity rise by $24 \mathrm{~h}$ after the injury and remain elevated through P2, approximately 6 days after the insult.

\section{Serum KCC2 Degradation Product Levels}

KCC2 levels rise over a critical period of cortical circuit development $[18,27]$, with the predominant increase in the cerebrum in the second postnatal week $[22,28]$. Because calpain degrades KCC2 into $90-\mathrm{kDa}$ fragments [19, 20], we hypothesized that the KCC2 fragments may be detectable in serum as a surrogate marker of cerebral neu- rodevelopment and injury. Serum levels of KCC2 90-kDa degradation fragments were elevated in serum from P11 TSHI-veh rats $(\mathrm{n}=13)$ compared to shams $(\mathrm{n}=10$, twoway ANOVA, $\mathrm{p}=0.02$ ) and were normalized in TSHI$\mathrm{EPO}$ rats that had received postnatal EPO treatment $(\mathrm{n}=$ $11, \mathrm{p}<0.001$; fig. $6 \mathrm{~d}$ ), mirroring the decreased oligomeric KCC2 expression found in the brain at the same time point. Together, these results suggest that serum $90-\mathrm{kDa}$ KCC2 fragments may serve as a biomarker of injury to the developing cerebrum and of responsiveness to successful treatment with a neuroprotective agent.

\section{Discussion}

Signs of excess CNS calpain activity have been found in both preclinical [23] and clinical studies of pediatric traumatic brain injury [29]. In rat models of term birth perinatal brain injury, excess calpain activity was present for $>48 \mathrm{~h}$ after P7 HI injury [30-32] and 5 days after P10 neonatal stroke [33]. Using an in vitro model of oxygenglucose deprivation injury at P7, the addition of EPO inhibited calpain activity [34]. Calpain activity and its responsiveness to EPO treatment have not previously been investigated in vivo in a model that mimics encephalopathy of prematurity. Here, we found evidence of both excess serum and cerebral calpain activity in vivo over an extended postnatal period, using an established model of CNS injury from preterm birth induced in rats by TSHI on E18 $[8,35]$. Serum calpain activity measured by aIISDP fragment levels was elevated $24 \mathrm{~h}$ after the E18 insult, remained increased through $\mathrm{P} 2$, and normalized by $\mathrm{P} 7$. 
Interestingly, prenatal TSHI induces a marked elevation of cerebral calpain activity, as shown by an increase in the ratio of cleaved to full-length aII-SDP in the cerebral cortex beginning at P2, 6 days after injury. The excess cerebral calpain activity is sustained through at least P11, 2 weeks following prenatal TSHI, and approximately equal to slightly after full term in humans. The extended period of excess cerebral calpain activity correlates with the protracted period of apparent CNS injury in encephalopathy of prematurity [36, 37]. To our knowledge, this is the first demonstration that excess calpain activity is present in a model of encephalopathy of prematurity, and that degradation products can be assayed in postnatal serum.

The pattern of CNS injury observed in preterm infants is complex and multifactorial [1]. It involves injury to both white and gray matter and encompasses injury to immature oligodendrocytes, axons, neurons and developing circuits over a sustained portion of a critical window of neurodevelopment. Developing white matter is commonly assayed biochemically through the investigation of MBP, and axonal integrity is examined via the ratio of phosphorylated to total NF levels [35]. In gray matter, the excitatory/inhibitory balance appears particularly sensitive to third-trimester injury [38]. In multiple developing cerebral cortical regions, the upregulation of membrane-bound KCC2 expression determines the intracellular chloride concentration. In a simplified view, increasing KCC2 chloride extrusion leads to the transition from a depolarizing to a hyperpolarizing response to GABA ( $\gamma$-amino butyric acid) activation of $\mathrm{GABA}_{\mathrm{A}}$ and glycine receptors [27]. A loss of KCC2 expression was found in postmortem cerebral samples from human preterm infants with white matter lesions [39]. In a preclinical model, prenatal global HI injury reduced KCC2 expression in the hippocampal CA3 subfield [18], and premature loss of subplate from this injury caused KCC2 loss in cerebral cortical layer IV [22]. Here, we show that cerebral levels of known calpain substrates that are also key neurodevelopmental molecules, including KCC2, MBP and NF, are reduced for an extended postnatal period through P11, consistent with prior reports that excess calpain activity contributes to KCC2 loss in the hippocampal CA3 subfield [18]. While the reduction in MBP and NF expression following prenatal injury is likely to be multifactorial, here we show that the reduced expression of $\mathrm{MBP}$ and $\mathrm{pNF} / \mathrm{NF}$ ratio also correlates with the excess calpain activity.

EPO is an emerging neuroprotective agent with multiple mechanisms of action that is currently being tested in the PENUT trial (NCT01378273) in infants born prior to 28 weeks gestation. Using a postnatal EPO dosing regimen that has previously produced functional improvement following prenatal TSHI $[9,22]$ and that is similar to the dosing regimen in the PENUT trial, we found that EPO treatment from P1 to P5 significantly modulated cerebral calpain activity at P11. Postnatal EPO treatment also restored levels of $\mathrm{MBP}$ and the $\mathrm{pNF} / \mathrm{NF}$ ratio at $\mathrm{P} 11$ and $\mathrm{P} 28$. While EPO has previously been shown to reduce excess calpain activity in the mature CNS [26], this is the first time that EPO modulation of excess calpain activity in the developing brain in vivo has been reported.

To further elucidate the potential mechanisms of EPO neuroprotection in the last trimester, we investigated the role of excess calpain activity and the degradation of molecules essential to white and gray matter function. Unbound EPO receptors drive neural cells to apoptosis as part of the normal neurodevelopmental regulation of neuronal number [40-42], and prenatal TSHI induces a marked upregulation of EPO receptor expression in the CNS without concomitant adequate ligand expression [9, 35]. Prenatal TSHI causes excess cerebral caspase- 3 activation [8], and that is mitigated by postnatal EPO treatment [9]. While excess caspase and calpain activation have been implicated in models of term birth CNS injury $[30,31,33,43]$, calpain activation or its modulation has not been previously explored in vivo in a preclinical model of encephalopathy of prematurity. Excess calpain activity is associated with a sustained reduction in the cerebral expression of molecules important for neurological development and calpain targets, including the co-transporter KCC2, MBP and NF. Notably, the reduced expression of these molecules was restored by postnatal treatment with EPO. The loss of these proteins is not solely attributable to excess calpain activity and probably varies depending on the molecule. For example, KCC2 levels are quite sensitive to calpain degradation mediated through calcium activation of NMDA receptors [18-20]. By contrast, numerous stages in the development of oligodendrocytes are impacted by prenatal TSHI, including oligodendrogenesis, survival and process extension [8,44], and EPO treatment reverses these deficits [44], suggesting that calpain degradation of myelin is only one component of myelin deficiency of encephalopathy of prematurity. Excess calpain degradation contributes to NF loss in preclinical models of traumatic injury and multiple sclerosis, and calpastatin overexpression or calpain inhibition restores the loss [12, 17, 45-47]. Calpain inhibition has also restored neurological function in preclinical in vivo models of Alzheimer's disease, tauopathy and Parkinson's disease [48-50]. Importantly, calpain inhibition 
improves functional outcomes in neurodevelopmental processes, including lissencephaly [51] and spinocerebellar ataxia type 3 (Machado-Joseph disease) [52]. Together, the progress in these other disease processes and our demonstration of the EPO-induced reduction of cerebral calpain activity and the associated loss of critical CNS developmentally regulated molecules suggest that the modulation of calpain activity over a sustained period may be beneficial to long-term neurological outcomes in neonates with encephalopathy of prematurity.

The mechanism of EPO-induced reduction in calpain activity in this model of encephalopathy of prematurity is not yet known. Because activated caspases cleave calpastatin $[53,54]$, and here the reduced levels of cerebral calpastatin expression were restored with EPO treatment, one potential mechanism for the effect of EPO on calpain activity may be by reducing calpastatin degradation and restoring the balance of calpain and calpastatin activities. Alternatively, EPO treatment may be affecting apoptosis and subsequent calpain activation through another pathway unrelated to calpastatin. For example, D'Orsi et al. [55] showed that calpain activation occurs downstream of mitochondrial engagement during excitotoxic apoptosis. Additionally, in Alzheimer's disease $\mu$-calpain activates GSK-3 $\beta$ [56]. Because EPO signaling modulates kinase activity, including activating Akt and inhibiting GSK-3 $\beta$ [57], it may be impacting calpain activity by altering the balance of Akt/GSK-3 $\beta$ activation. Another possibility involves an imbalance of vascular endothelial growth factor signaling after injury that triggers calpain activation and is modulated by EPO treatment, similar to the mechanism suggested in a preclinical model of cerebral malaria [26]. In a nonhuman primate model of neonatal asphyxia, the addition of EPO treatment to postinjury hypothermia significantly improved chronic behavioral outcomes, which suggests that EPO can synergize with other neuroprotective strategies, perhaps through the modulation of calpain activation [58]. Clarifying the details of the mechanism is beyond the scope of this work but is being actively investigated. In addition to directly limiting calpain activity, EPO may counteract the detrimental effects of excess calpain activity that limit neurorepair. For example, after stroke in the mature CNS, excess calpain activity hinders neural stem and neuroblast proliferation and migration [59]. EPO signaling promotes multiple stages of neuronal and oligodendroglial development $[44,60]$ and thus may counterbalance calpain-induced suppression of repair. The pattern of sustained elevation of excess calpain activity found here is similar to the sustained elevation of caspase-3 activity previously observed in this model $[8,9]$ and may contribute to the sustained period of persistent lack of recovery that has been implicated in human preterm infants [36, 37].

Elevated cerebral calpain activity is reflected in serum in this model of encephalopathy of prematurity. Here we have presented three novel findings related to the serum levels. To our knowledge, this is the first time that the elevation of serum aII-SDP levels has been shown with CNS calpain activity in vivo in a clinically relevant model of encephalopathy of prematurity. This suggests the possibility that serum biomarkers could be used not only to diagnose CNS injury in the neonatal period, but also to guide the use of neuroprotective interventions such as EPO. For example, preterm infants with CNS insults are prone to cumulative injuries from additional postnatal problems such as sepsis and seizures, and serum biomarkers could guide the duration of treatment for CNS protection. The need for serum biomarkers for preterm infants, as well as infants with other perinatal CNS insults, is well recognized [61-63].

Finally, calpain-mediated KCC2 degradation products were also found in serum after prenatal TSHI and appeared responsive to EPO treatment. Cerebral KCC2 expression rises significantly in the second postnatal week [64]. Calpain cleaves KCC2 into a 90-kDa fragment [18-20], which prompted us to investigate whether serum would reflect ongoing CNS injury in the postnatal period and potential restoration with EPO treatment. Sustained excess CNS calpain activity following prenatal TSHI is likely to contribute to the prolonged period of impaired neurodevelopment observed in extremely preterm infants. Moreover, postnatal EPO treatment modulated $90-\mathrm{kDa}$ KCC2 levels after prenatal injury, with restoration back to sham levels. These results suggest that KCC2 calpain fragments can be assayed in serum following perinatal CNS injury, and that they may prove useful as CNS injury biomarkers of both injury and EPO responsiveness. Given the importance of KCC2 expression to neuronal health [27], serum KCC2 fragments offer a promising line of investigation for future study. Because $90-\mathrm{kDa}$ levels are currently only detectable with immunoblotting to separate fragments, additional assay development is needed prior to widespread clinical utility and is already underway. Patterns of serum biomarker trends may be more informative than a single marker [65] and provide a less invasive means to monitor the type, extent and duration of CNS injury in critically ill infants who are too ill to undergo detailed high-resolution imaging studies. 


\section{Acknowledgments}

This study was funded by the National Institutes of Neurological Disorders and Stroke (NINDS) at the National Institutes of Health (NIH; R01 NS060765 to S.R.) and by the Boston Children's Hospital Translational Research Program (pilot funding to S.R. and L.L.J.).

\section{Disclosure Statement}

The authors declare no conflicts of interest.

\section{References}

1 Volpe J: Brain injury in premature infants: a complex amalgam of destructive and developmental disturbances. Lancet Neurol 2009; 8:110-124.

2 Wang KK: Calpain and caspase: can you tell the difference? By Kevin K.W. Wang Vol 23, pp 20-26. Trends Neurosci 2000;23:59.

3 Robinson S, Li Q, DeChant A, Cohen M: Neonatal loss of $\gamma$-amino butyric acid pathway expression after human perinatal brain injury. J Neurosurg 2006;104:396-408.

-4 Chamnanvanakij S, Margraf LR, Burns D, Perlman JM: Apoptosis and white matter injury in preterm infants. Pediatr Dev Pathol 2002;5:184-189.

5 Hargitai B, Szabo V, Hajdu J, Harmath A, Pataki M, Farid P, Papp Z, Szende B: Apoptosis in various organs of preterm infants: histopathologic study of lung, kidney, liver, and brain of ventilated infants. Pediatr Res 2001; 50:110-114.

-6 Felderhoff-Mueser U, Buhrer C, Groneck P, Obladen M, Bartmann P, Heep A: Soluble Fas (CD95/Apo-1), soluble Fas ligand, and activated caspase 3 in the cerebrospinal fluid of infants with posthemorrhagic and nonhemorrhagic hydrocephalus. Pediatr Res 2003;54: 659-664.

7 Digicaylioglu M, Lipton SA: Erythropoietinmediated neuroprotection involves cross-talk between Jak2 and NF- $\kappa B$ signalling cascades. Nature 2001;412:641-647.

8 Robinson S, Petelenz K, Li Q, Cohen M, Buczek M, Lust D, Miller R: Developmental changes induced by prenatal hypoxia-ischemia insult in rats models human perinatal brain injury. Neurobiol Dis 2005; 18:568-581.

-9 Mazur M, Miller R, Robinson S: Postnatal erythropoietin treatment mitigates neural cell loss after systemic prenatal hypoxic-ischemic injury. J Neurosurg Pediatr 2010;6:206-221.

10 Li Y, Bondada V, Joshi A, Geddes JW: Calpain 1 and calpastatin expression is developmentally regulated in rat brain. Exp Neurol 2009; 220:316-319.

- 11 Zadran S, Akopian G, Zadran H, Walsh J, Baudry M: RVG-mediated calpain2 gene silencing in the brain impairs learning and memory. Neuromolecular Med 2013;15:74-81.

-12 Yang J, Weimer RM, Kallop D, Olsen O, Wu Z, Renier N, Uryu K, Tessier-Lavigne M: Regulation of axon degeneration after injury and in development by the endogenous calpain inhibitor calpastatin. Neuron 2013;80:1175-1189.
13 Nath R, Raser KJ, Stafford D, Hajimohammadreza I, Posner A, Allen H, Talanian RV, Yuen P, Gilbertsen RB, Wang KK: Non-erythroid alpha-spectrin breakdown by calpain and interleukin 1 beta-converting-enzymelike protease(s) in apoptotic cells: contributory roles of both protease families in neuronal apoptosis. Biochem J 1996;319:683-690.

14 Saatman KE, Creed J, Raghupathi R: Calpain as a therapeutic target in traumatic brain injury. Neurotherapeutics 2010;7:31-42.

15 Jadhav S, Zilka N, Novak M: Protein truncation as a common denominator of human neurodegenerative foldopathies. Mol Neurobiol 2013;48:516-532.

16 Vosler PS, Brennan CS, Chen J: Calpain-mediated signaling mechanisms in neuronal injury and neurodegeneration. Mol Neurobiol 2008;38:78-100.

17 Das A, Guyton MK, Smith A, Wallace GT, McDowell ML, Matzelle DD, Ray SK, Banik NL: Calpain inhibitor attenuated optic nerve damage in acute optic neuritis in rats. J Neurochem 2013;124:133-146.

18 Jantzie LL, Getsy PM, Firl DG, Wilson CG, Miller RH, Robinson S: Erythropoietin attenuates loss of potassium chloride co-transporters following prenatal brain injury. Mol Cell Neurosci 2014;61:152-162.

19 Puskarjov M, Ahmad F, Blaesse P: Activitydependent cleavage of the $\mathrm{K}-\mathrm{Cl}$ cotransporter KCC2 mediated by calcium-activated protease calpain. J Neurosci 2012;32:11356-11364.

20 Zhou H, Chen S, Byun H, Chen H-X, Li L, Han H, Lopez-Berestein G, Sood A, Pan H: $\mathrm{N}$-methyl-D-aspartate receptor- and calpainmediated proteolytic cleavage of $\mathrm{K}^{+}-\mathrm{Cl}^{-} \mathrm{co}-$ transporter-2 impairs spinal chloride homeostasis in neuropathic pain. J Biol Chem 2012; 287:33853-33864.

21 Ma M: Role of calpains in the injury-induced dysfunction and degeneration of the mammalian axon. Neurobiol Dis 2013;60:61-79.

22 Jantzie LL, Corbett CJ, Firl DJ, Robinson S: Postnatal erythropoietin mitigates impaired cerebral cortical development following subplate loss from prenatal hypoxia-ischemia. Cereb Cortex 2015;25:2683-2695.

23 Schober ME, Requena DF, Davis LJ, Metzger RR, Bennett KS, Morita D, Niedzwecki C, Yang Z, Wang KK: Alpha II spectrin breakdown products in immature Sprague Dawley rat hippocampus and cortex after traumatic brain injury. Brain Res 2014;1574:105-112.
24 Liu MC, Akle V, Zheng W, Dave JR, Tortella FC, Hayes RL, Wang KK: Comparing calpainand caspase-3-mediated degradation patterns in traumatic brain injury by differential proteome analysis. Biochem J 2006;394:715-725.

25 Sato K, Minegishi S, Takano J, Plattner F, Saito T, Asada A, Kawahara H, Iwata N, Saido TC, Hisanaga S: Calpastatin, an endogenous calpain-inhibitor protein, regulates the cleavage of the Cdk5 activator p35 to p25. J Neurochem 2011;117:504-515

26 Hempel C, Hoyer N, Staalso T, Kurtzhals JA: Effects of the vascular endothelial growth factor receptor-2 (VEGFR-2) inhibitor SU5416 on in vitro cultures of Plasmodium falciparum. Malar J 2014;13:201.

27 Kaila K, Price TJ, Payne JA, Puskarjov M, Voipio J: Cation-chloride cotransporters in neuronal development, plasticity and disease. Nat Rev Neurosci 2014;15:637-654.

28 Daw M, Ashby M, Issac J: Coordinated developmental recruitment of latent fast spiking interneurons in layer IV barrel cortex. Nat Neurosci 2007;10:453-461.

29 Berger RP, Hayes RL, Richichi R, Beers SR, Wang KK: Serum concentrations of ubiquitin C-terminal hydrolase-L1 and aII-spectrin breakdown product $145 \mathrm{kDa}$ correlate with outcome after pediatric TBI. J Neurotrauma 2012;29:162-167.

- 30 Blomgren K, McRae A, Bona E, Saido TC Karlsson JO, Hagberg H: Degradation of fodrin and MAP 2 after neonatal cerebral hypoxic-ischemia. Brain Res 1995;684:136-142.

31 Blomgren K, Zhu C, Wang X, Karlsson JO, Leverin AL, Bahr BA, Mallard C, Hagberg H: Synergistic activation of caspase- 3 by $m$-calpain after neonatal hypoxia-ischemia: a mechanism of 'pathological apoptosis'? J Biol Chem 2001;276:10191-10198.

32 Rosenkranz K, May C, Meier C, Marcus K: Proteomic analysis of alterations induced by perinatal hypoxic-ischemic brain injury. J Proteome Res 2012;11:5794-5803.

33 Shimotake J, Derugin V, Wendland M, Vexler ZS, Ferriero DM: Vascular endothelial growth factor receptor-2 inhibition promotes cell death and limits endothelial cell proliferation in a neonatal rodent model of stroke. Stroke 2010;41:343-349.
EPO Modulates Calpain Activity

following Prenatal HI
Dev Neurosci 2016;38:15-26 DOI: $10.1159 / 000441024$ 
34 Montero M, Poulsen FR, Noraberg J, Kirkeby A, van Beek J, Leist M, Zimmer J: Comparison of neuroprotective effects of erythropoietin (EPO) and carbamylerythropoietin (CEPO) against ischemia-like oxygen-glucose deprivation (OGD) and NMDA excitotoxicity in mouse hippocampal slice cultures. Exp Neurol 2007;204:106-117.

- 35 Jantzie LL, Corbett CJ, Berglass J, Firl DJ, Flores J, Mannix R, Robinson S: Complex pattern of interaction between in utero hypoxiaischemia and intra-amniotic inflammation disrupts brain development and motor function. J Neuroinflammation 2014;11:131.

- 36 O'Shea TM, Allred EN, Kuban KC, Dammann O, Paneth N, Fichorova R, Hirtz D, Leviton A; Extremely Low Gestational Age Newborn (ELGAN) Study Investigators: Elevated concentrations of inflammation-related proteins in postnatal blood predict severe developmental delay at 2 years of age in extremely preterm infants. J Pediatr 2012;160: 395-401.e4.

-37 Dammann O, Leviton A: Intermittent or sustained systemic inflammation and the preterm brain. Pediatr Res 2014;75:376-380.

- 38 Robinson S: Systemic prenatal insults disrupt telencephalon development: Implications for treatment. Epilepsy Behav 2005;7:345-363.

-39 Robinson S, Mikolaenko I, Thompson I, Cohen M, Goyal M: Loss of cation-chloride cotransporter expression in preterm infants with white matter lesions: implications for the pathogenesis of epilepsy. J Neuropath Exp Neurol 2010;69:565-572.

-40 Yu X, Shacka J, Eells J, Suarez-Quian C, Przygodzki R, Beleslin-Cokic B, Lin C-S, Nikodem V, Hempstead B, Flanders K, Costantini F, Noguchi C: Erythropoietin receptor signalling is required for normal brain development. Development 2002;129:505-516.

-41 Knabe W, Siren A-L, Ehrenreigh H, Kuhn H-J: Expression patterns of erythropoietin and its receptor in the developing spinal cord and dorsal root ganglia. Anat Embryol 2005; 210:209-219.

-42 Knabe W, Knerlich F, Washausen S, Kietzmann T, Siren A, Brunnett G, Kuhn H, Ehrenreich $\mathrm{H}$ : Expression patterns of erythropoietin and its receptor in the developing midbrain. Anat Embryol 2004;207:503-512.

-43 Sheldon RA, Sadjadi R, Lam M, Fitzgerald R, Ferriero DM: Alteration in downstream hypoxia gene signaling in neonatal glutathione peroxidase overexpressing mouse brain after hypoxia-ischemia. Dev Neurosci 2015; 37:398-406.

-44 Jantzie LL, Miller RH, Robinson RH: Erythropoietin signaling promotes oligodendrocyte development following prenatal systemic hypoxic-ischemic brain injury. Pediatr Res 2013;74:658-667.
45 Ma M, Shofer FS, Neumar RW: Calpastatin overexpression protects axonal transport in an in vivo model of traumatic axonal injury. J Neurotrauma 2012;29:2555-2563.

46 Schoch KM, Evans HN, Brelsfoard JM, Madathil SK, Takano J, Saido TC, Saatman KE: Calpastatin overexpression limits calpain-mediated proteolysis and behavioral deficits following traumatic brain injury. Exp Neurol 2012;236:371-382.

47 Ma M, Ferguson TA, Schoch KM, Li J, Qian Y, Shofer FS, Saatman KE, Neumar RW: Calpains mediate axonal cytoskeleton disintegration during Wallerian degeneration. Neurobiol Dis 2013;56:34-46.

48 Trinchese F, Fa M, Liu S, Zhang H, Hidalgo A, Schmidt SD, Yamaguchi $\mathrm{H}$, Yoshii N, Mathews PM, Nixon RA, Arancio O: Inhibition of calpains improves memory and synaptic transmission in a mouse model of Alzheimer disease. J Clin Invest 2008;118:27962807.

49 Rao MV, McBrayer MK, Campbell J, Kumar A, Hashim A, Sershen H, Stavrides PH, Ohno M, Hutton M, Nixon RA: Specific calpain inhibition by calpastatin prevents tauopathy and neurodegeneration and restores normal lifespan in tau P301L mice. J Neurosci 2014; 34:9222-9234.

50 Diepenbroek M, Casadei N, Esmer H, Saido TC, Takano J, Kahle PJ, Nixon RA, Rao MV, Melki R, Pieri L, Helling S, Marcus K, Krueger R, Masliah E, Riess O, Nuber S: Overexpression of the calpain-specific inhibitor calpastatin reduces human alpha-synuclein processing, aggregation and synaptic impairment in $[\mathrm{A} 30 \mathrm{P}]$ aSyn transgenic mice. Hum $\mathrm{Mol}$ Genet 2014;23:3975-3989.

51 Toba S, Tamura Y, Kumamoto K, Yamada M, Takao K, Hattori S, Miyakawa T, Kataoka Y, Azuma M, Hayasaka K, Amamoto M, Tominaga $\mathrm{K}$, Wynshaw-Boris $\mathrm{A}$, Wanibuchi $\mathrm{H}$, Oka Y, Sato M, Kato M, Hirotsune S: Postnatal treatment by a blood-brain-barrier permeable calpain inhibitor, SNJ1945, rescued defective function in lissencephaly. Sci Rep 2013;3:1224.

52 Simoes AT, Goncalves N, Nobre RJ, Duarte CB, Pereira de Almeida L: Calpain inhibition reduces ataxin-3 cleavage alleviating neuropathology and motor impairments in mouse models of Machado-Joseph disease. Hum Mol Genet 2014;23:4932-4944.

53 Wang KK, Posmantur R, Nadimpalli R, Nath R, Mohan P, Nixon RA, Talanian RV, Keegan M, Herzog L, Allen H: Caspase-mediated fragmentation of calpain inhibitor protein calpastatin during apoptosis. Arch Biochem Biophys 1998;356:187-196.

54 Porn-Ares MI, Samali A, Orrenius S: Cleavage of the calpain inhibitor, calpastatin, during apoptosis. Cell Death Differ 1998;5:10281033.
55 D’Orsi B, Bonner H, Tuffy LP, Dussmann H, Woods I, Courtney MJ, Ward MW, Prehn JH: Calpains are downstream effectors of bax-dependent excitotoxic apoptosis. J Neurosci 2012;32:1847-1858.

-56 Jin N, Yin X, Yu D, Cao M, Gong CX, Iqbal K, Ding F, Gu X, Liu F: Truncation and activation of GSK-3 $\beta$ by calpain I: a molecular mechanism links to tau hyperphosphorylation in Alzheimer's disease. Sci Rep 2015;5: 8187.

57 Shang Y, Wu Y, Yao S, Wang X, Feng D, Yang W: Protective effect of erythropoietin against ketamine-induced apoptosis in cultured rat cortical neurons: involvement of PI3K/Akt and GSK-3 beta pathway. Apoptosis 2007;12: 2187-2195.

58 Traudt CM, McPherson RJ, Bauer LA, Richards TL, Burbacher TM, McAdams RM, Juul SE: Concurrent erythropoietin and hypothermia treatment improve outcomes in a term nonhuman primate model of perinatal asphyxia. Dev Neurosci 2013;35:491-503.

59 Machado VM, Morte MI, Carreira BP, Azevedo MM, Takano J, Iwata N, Saido TC, Asmussen H, Horwitz AR, Carvalho CM, Araujo IM: Involvement of calpains in adult neurogenesis: implications for stroke. Front Cell Neurosci 2015;9:22.

60 Iwai M, Cao CM, Yin W, Stetler RA, Liu J, Chen J: Erythropoietin promotes neuronal replacement through revascularization and neurogenesis after neonatal hypoxia/ischemia in rats. Stroke 2007;38:2795-2803.

61 Massaro AN, Jeromin A, Kadom N, Vezina G, Hayes RL, Wang KK, Streeter J, Johnston MV: Serum biomarkers of MRI brain injury in neonatal hypoxic ischemic encephalopathy treated with whole-body hypothermia: a pilot study. Pediatr Crit Care Med 2013;14:310317.

62 Douglas-Escobar M, Weiss MD: Biomarkers of brain injury in the premature infant. Front Neurol 2012;3:185.

63 Chun PT, McPherson RJ, Marney LC, Zangeneh SZ, Parsons BA, Shojaie A, Synovec RE, Juul SE: Serial plasma metabolites following hypoxic-ischemic encephalopathy in a nonhuman primate model. Dev Neurosci 2015; 37:161-171.

64 Dzhala V, Talos D, Sdrulla D, Brumback A Mathews G, Benke T, Delpire E, Jensen F, Staley K: NKCC1 transporter facilitates seizures in the developing brain. Nat Med 2005; 11:1205-1213.

65 Berger RP, Bazaco MC, Wagner AK, Kochanek PM, Fabio PM: Trajectory analysis of serum biomarker concentrations facilitates outcome prediction after pediatric traumatic and hypoxemic brain injury. Dev Neurosci 2010; 32:396-405. 